\title{
VPg of murine norovirus binds translation initiation factors in infected cells
}

\author{
Katie F Daughenbaugh ${ }^{1}$, Christiane E Wobus ${ }^{2}$ and Michele E Hardy*1
}

Address: ${ }^{1}$ Veterinary Molecular Biology, Montana State University, Bozeman, MT, USA and ${ }^{2}$ Department of Pathology and Immunology, Washington University School of Medicine, St Louis, MO, USA

Email: Katie F Daughenbaugh - katief@montana.edu; Christiane E Wobus - cwobus@wustl.edu; Michele E Hardy* - mhardy@montana.edu

* Corresponding author

Published: 23 May 2006

Virology Journal 2006, 3:33 doi:10.1186/1743-422X-3-33

This article is available from: http://www.virologyj.com/content/3/I/33

(c) 2006 Daughenbaugh et al; licensee BioMed Central Ltd.

This is an Open Access article distributed under the terms of the Creative Commons Attribution License (http://creativecommons.org/licenses/by/2.0), which permits unrestricted use, distribution, and reproduction in any medium, provided the original work is properly cited.
Received: 06 March 2006

Accepted: 23 May 2006

\begin{abstract}
Background: Norovirus genomic and subgenomic RNAs are covalently linked at the 5 ' nucleotide to a $15 \mathrm{kD}$ protein called $\mathrm{VPg}$. VPg of two human norovirus strains binds translation initiation factor elF3 and other elFs in vitro, suggesting VPg functions in initiation of protein synthesis on viral RNA. Human norovirus strains are not cultivable, and thus experimental evidence of interactions between VPg and elFs in infected cells has been lacking. We used the cultivable murine norovirus MNV-I as a model to study interactions between VPg and elFs in infected cells.
\end{abstract}

Results: As shown previously for human norovirus VPg, MNV-I VPg bound elF3, elF4GI, elF4E, and S6 ribosomal protein in cell extracts by GST pull-down assay. Importantly, MNV-I VPg coprecipitated elF4GI and elF4E from infected macrophages, providing evidence that VPg interacts with components of the translation machinery in norovirus infected cells.

Conclusion: The interactions between MNV-I VPg and elFs completely mimic those reported for the human norovirus VPg, illustrating the utility of MNV-I as a relevant molecular model to study mechanisms of human norovirus replication.

\section{Background}

Noroviruses constitute a genus in the family Caliciviridae and are the most frequent cause of foodborne viral gastroenteritis epidemics [1]. The norovirus genome is a $7.7 \mathrm{~kb}$, positive-sense, single-stranded RNA that is polyadenylated at the 3' end. The genome codes for three open reading frames. ORF1 encodes the nonstructural proteins that are synthesized as a polyprotein precursor and processed by the 3C-like viral protease. ORF2 and ORF3 encode the capsid protein VP1 and minor structural protein VP2, respectively. Both VP1 and VP2 are synthesized from a subgenomic RNA that is 3 ' coterminal with the genomic RNA. Instead of a 7-methylguanosine $\left(\mathrm{m}^{7} \mathrm{G}\right)$ cap structure at the $5^{\prime}$ end, genomic and subgenomic RNAs are covalently linked to a viral protein called $\mathrm{VPg}$, for viral protein genome-linked. The linkage between VPg and the 5 ' nucleotide has been demonstrated experimentally only for the animal caliciviruses [2,3], but by analogy, it is assumed that VPg also is linked to norovirus genomes. The function(s) of VPg in the norovirus replication cycle are not known, but data that this small protein functions in translation initiation are accumulating. Studies of animal caliciviruses showed VPg is necessary for infectivity of native viral RNA [2], but an $\mathrm{m}^{7} \mathrm{G}$ cap can confer infectivity to in vitro synthesized feline calicivirus (FCV) genomic RNA transcripts [4]. Together, data from these studies conclude that the $\mathrm{m}^{7} \mathrm{G}$ cap functionally substitutes for $\mathrm{VPg}$ when transfecting calicivirus RNA into cells. We previ- 


\begin{abstract}
MNV - 1 GK-KGKNKKGRGRPG---VFRTRGLTDEEYDEFKKRRESRGGKYSIDDYLADRERNV GKNKGKTKKGRGRKNNYNAFSRRGLSDEEYEEYKKIREEKNGNYSIQEYLEDRQRY

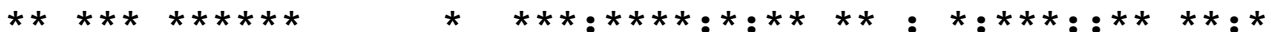

MNV-1 EEELLERDE---------EEAIFGDGFGLKATRRSRKAERAKLGLVSGGDIRARKPIDWN NV EEELAEVQAGGDGGIGETEME I RHRVFYKSKSKKHQQEQRRQLGLVTGSDIRKRKPIDWT $* * * * *$ : * *

* $::::: *: * * * *: * * * * * * * * * *$

MNV-1 VVGPSWADDDRQVDYGEKINFE 994

NV PPKNEWADDDREVDYNEKINFE 1100

$\star \star \star * * * * * * * * * * * *$

MNV-I VPg and NV VPg share significant amino acid sequence identity. Sequences encoding MNV-I VPg and NV VPg were aligned with ClustalW. Asterisks indicate identical residues. Semicolons are conservative substitutions. Amino acid numbers correspond to the position in the ORFI polyprotein.
\end{abstract}

\title{
Figure I
}

ously reported that VPg of the Norwalk (NV) and Snow Mountain (SMV) strains of human norovirus bound translation initiation factor eIF3, providing the first direct evidence that VPg may function in ribosome recruitment to viral RNA [5]. Recently, an interaction between FCV VPg and translation initiation factor eIF4E was reported, and this interaction was required for translation of FCV VPg-linked RNA in vitro [6].

Most cellular mRNAs are translated by a cap-dependent mechanism driven by protein-protein and protein-RNA interactions between translation initiation factors (eIFs) and mRNA [7]. Cap-dependent translation initiates with binding of the eIF4F complex that consists of eIF4E, eIF4GI and eIF4A, to the $\mathrm{m}^{7} \mathrm{G}$ cap structure. Recruitment of the 43S pre-initiation complex composed of eIF3, 40S ribosomal subunits, and initiator tRNA to mRNA occurs primarily through interactions between eIF4GI and eIF3 $[8,9]$. This $48 \mathrm{~S}$ complex scans the mRNA to initiate translation at the first strong context AUG. Cap-independent translation is mediated by extensively structured regions of RNA called internal ribosome entry sites (IRES). These elements are found in genomes of picornaviruses, pestiviruses, and insect viruses of the Dicistroviridae family [10], and some cellular mRNAs as well (reviewed in [11]. Initiation complexes assemble internal to the $5^{\prime}$ end of the RNA molecule and translation initiates at, or shortly downstream, of the IRES. The fact that norovirus RNAs lack a $\mathrm{m}^{7} \mathrm{G}$ cap, are $5^{\prime}$ nucleotide-protein linked, and have 5 ' untranslated regions (UTR) of only 10 nucleotides suggest translation initiation on norovirus RNA mediated by VPg proceeds in a manner distinct from cap-dependent and IRES-dependent mechanisms.

VPg of human noroviruses binds directly to eIF3, is present in complexes with other eIFs, and inhibits transla- tion of mRNAs that have different eIF requirements for functional initiation complex assembly [5]. All of these data were obtained using purified protein and cell-free assays. Human noroviruses do not grow in cell culture, and therefore, connecting this eIF binding data with events that occur in norovirus infected cells is challenging. A significant advance in the field was the discovery of a murine norovirus $(\mathrm{MNV}-1)$ that replicates in cultured macrophages and dendritic cells $[12,13]$. MNV-1 is the only norovirus that grows in culture, and is closely related genetically to the human norovirus strains [12]. Therefore, we used MNV-1 to address questions about the functional significance of interactions between VPg and eIFs observed with the human noroviruses. In addition, determining whether protein-protein interactions are the same between the human and murine viruses would establish whether MNV-1 constitutes a relevant molecular model for the non-cultivable human strains. Our data show that MNV-1 VPg binds eIFs in infected cells, and that interactions between MNV-1 VPg and eIFs are the same as those that occur between eIFs and human norovirus VPg. These findings indicate that $\mathrm{MNV}-1$ represents an important molecular tool for the study of human noroviruses in tissue culture.

\section{Results}

Recombinant MNV-I VPg binds elF3, elF4GI, elF4E, and S6 ribosomal protein in vitro

VPg of genogroup I NV and genogroup II SMV share $68 \%$ amino acid sequence identity and both bind purified eIF3 and to eIF3 in cell extracts [5]. VPg sequences of MNV-1 and NV were aligned and share 54\% identity (Figure 1). The $\mathrm{N}$ - and C-terminal domains showed the highest level of conservation, and flank a central variable region that includes a glycine-rich, nine amino acid insertion in $\mathrm{NV}$ VPg. Interpreted in the context of the inferred function of 
A

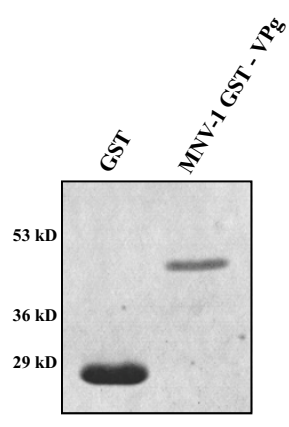

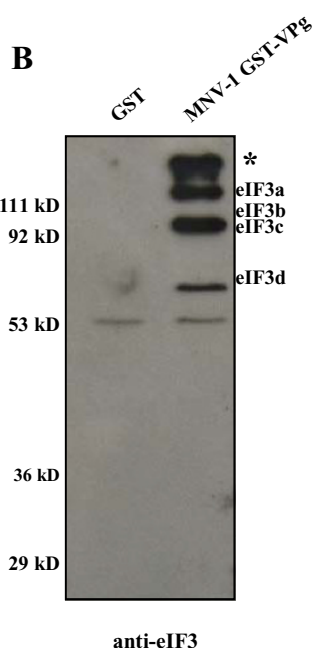
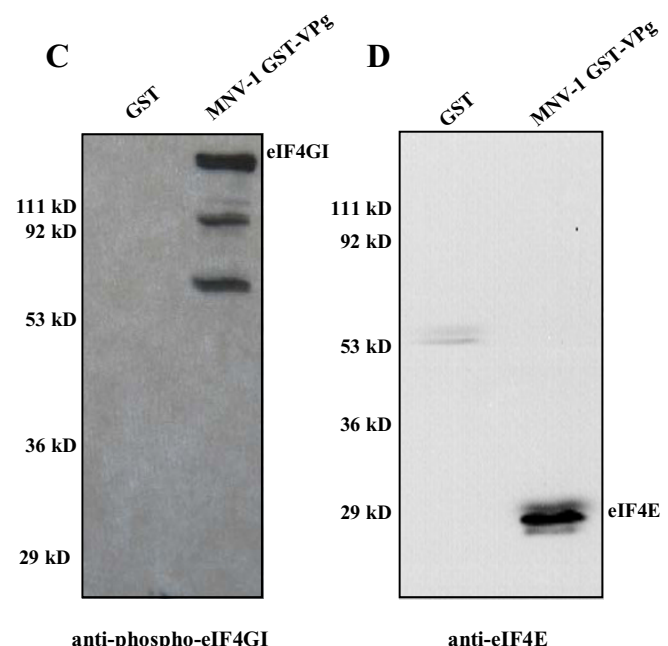

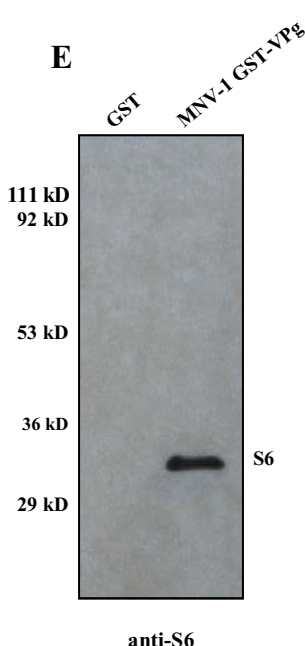

Figure 2

MNV-I GST-VPg binds elF3, phospho-elF4GI, elF4E, and S6 ribosomal protein by pull-down assay. GST and MNV-I GST-VPg were expressed in bacteria and purified as described in the text. A) Purified GST and GST-VPg, analyzed by $10 \%$ SDS-PAGE and stained with Coomassie blue. Proteins in the pull-down eluates were subjected to SDS-PAGE, and then immunoblot probed with (B) anti-elF3, (C) anti-phospho-elF4GI, (D) anti-elF4E, and (E) anti-S6 ribosomal protein. Blots were developed with ECL chemiluminescent substrate. The asterisk in B indicates a protein identified as elF4GI.

VPg in translation, an overall amino acid sequence identity of $54 \%$, and conservation in both $\mathrm{N}$ and C-terminal domains, suggested that MNV-1 could have similar eIF binding properties as NV VPg.

To test if MNV-1 VPg binds eIFs in a cell-free system, VPg was expressed as a GST fusion protein in bacteria, purified (Figure 2A), and used in pull-down assays with extracts prepared from RAW 264.7 macrophages as previously described [5]. Proteins in pull-down eluates were analyzed by immunoblots probed with antibodies to eIF3 (Figure 2B), phospho-eIF4GI (Figure 2C), eIF4E (Figure 2D), and S6 ribosomal protein (Figure 2E). Similar to data obtained for NV VPg, all of these factors bound MNV-1 GST-VPg.

eIF3 is a large complex composed of 12 subunits [7]. Four of these subunits, eIF3a, eIF3b/eIF3c doublet [14], and eIF3d, were identified in the VPg pull-down eluates. Identification was made by the banding pattern that is observed for eIF3 when this polyclonal antibody is used as a probe [14]. The reasons for detecting only a few of the subunits in the eluates are not known, but this large complex may be unstable under conditions of the assay. The anti-eIF3 serum contains antibodies to eIF4GI [14]. Therefore, the high molecular weight protein detected in the immunoblots probed with this serum likely represents eIF4GI. The presence of this factor in the pull-down reactions was confirmed by probing the blots with an antiphospho-eIF4GI antibody (Figure 2C). The lower molec- ular weight products that reacted with the phosphoeIF4GI antibody are interpreted to be degradation products of the full-length protein. Taken together, the data from the pull-down assays and previous results show that the interactions between NV VPg and eIFs and between MNV-1 VPg and eIFs are the same.

\section{VPg and elFs co-precipitate from MNV-I infected RAW 264.7 cells}

To investigate interactions between VPg and eIFs in infected cells, RAW 264.7 cells were infected with MNV-1 and immunoprecipitations (IP) were performed following established protocols $[13,15]$. MNV-1 VPg was present in infected but not mock infected cell lysate (Figure 3A, lane 2, middle panel) and antibodies to VPg immunoprecipitated VPg (Figure 3A, lane 4, middle panel) and phospho-eIF4GI (Figure 3A, lane 4, top panel). The polar nature of VPg results in a somewhat diffuse migration in SDS polyacrylamide gels instead of a sharp band (Daughenbaugh and Hardy, unpublished observations). In the reciprocal experiment, anti-phospho-eIF4GI antibody precipitated phospho-eIF4GI (Figure 3A, lanes 5 and 6, top panel) and MNV-1 VPg (Figure 3A, lane 6, middle panel). This demonstrates MNV-1 VPg binds eIF4GI in virus-infected cells.

Three additional observations about eIF4GI can be made from these experiments. First, there was a reproducible increase in the amount of phospho-eIF4GI in infected cells compared to mock infected cells (Figure 3A lanes 1, 


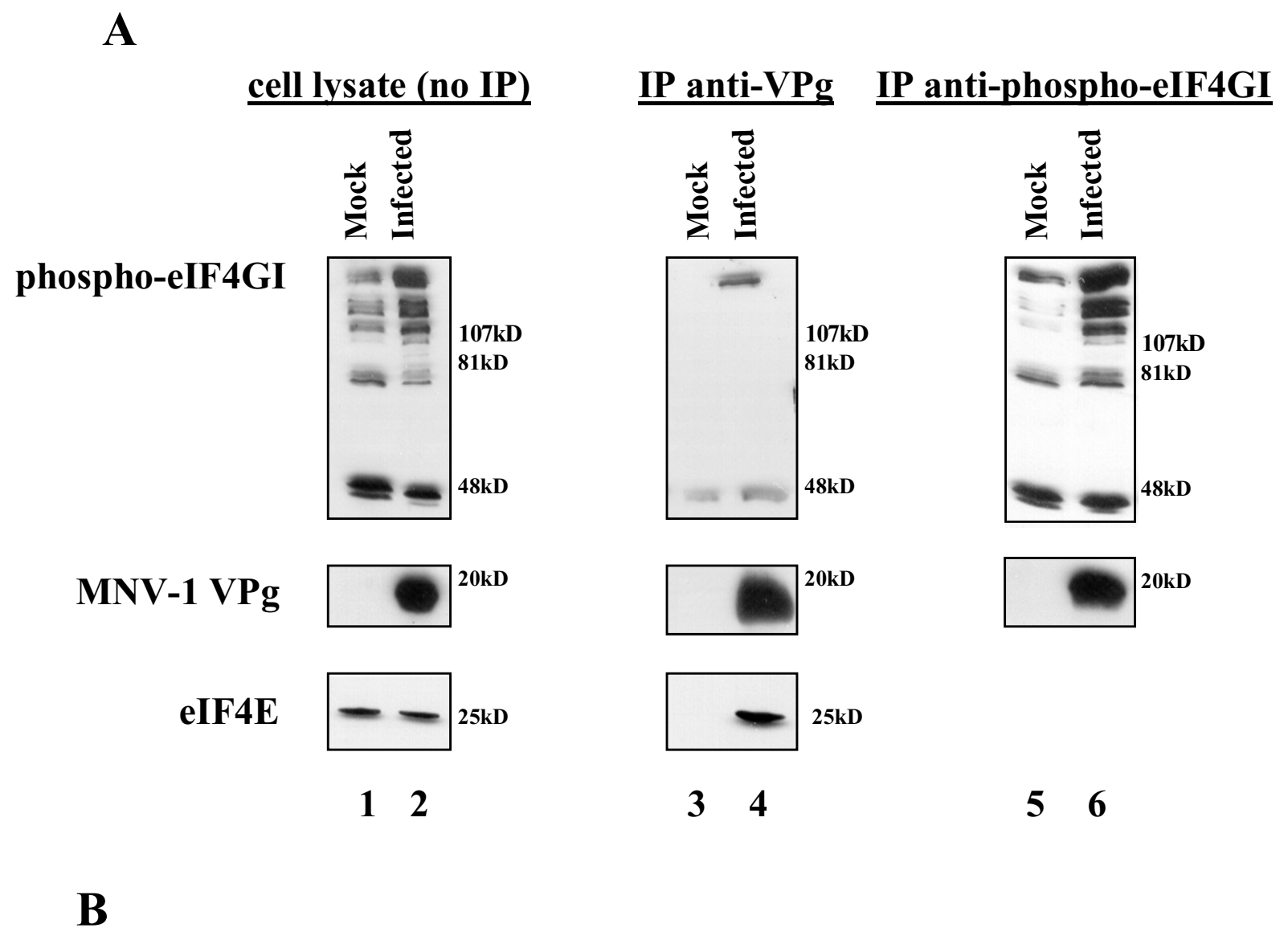

\section{cell lysate (no IP)}

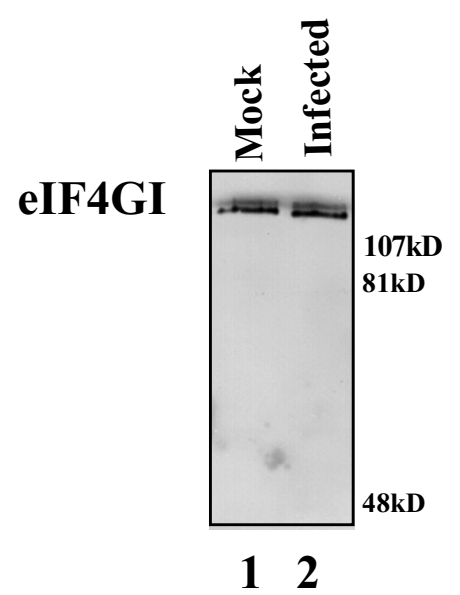

Figure 3

Phospho-elF4GI and elF4E co-immunoprecipitate with VPg from MNV-I infected RAW 264.7 cells. A) Mock infected or infected cell lysates were subjected to immunoprecipitation with anti-MNV-I VPg (lanes 3 and 4) or anti-phosphoelF4GI (lanes 5 and 6). Immunoblots were probed with the relevant antibody indicated to the left of the panels. Lanes I and 2 are control lysates that did not receive antibody. B) Mock infected or infected cell lysates probed with anti-elF4GI. 
2, 5 and 6, top panels). However, the levels of total eIF4GI, irrespective of its phosphorylation status, were similar in mock and infected cell lysates (Figure 3B) suggesting that differences in the amount of phospho-eIF4GI cannot be attributed to increased expression of eIF4GI. It has been reported that the amount of eIF4GI phosphorylated at serines 1108, 1148 and 1192 increases in response to serum stimulation, and phosphorylation at these sites is modulated by the $\mathrm{N}$ terminal third of the protein [16]. Potential mechanisms were proposed, including de-repression of eIF4GI by direct phosphorylation of the $\mathrm{N}$ terminus, or alternatively, interactions with an eIF4GI binding partner to expose the $\mathrm{C}$ terminal domain to kinase activity [16]. It is conceivable that MNV-1 infection could result in activation of kinases that modulate the activity of eIF4GI. An alternative and not mutually exclusive possibility is that direct interactions between eIF4GI and VPg alter the conformation of eIF4GI to a form where the $\mathrm{C}$ terminal serine residues are phosphorylated.

A second observation is that several eIF4GI degradation products were found in cell lysate (Figure 3A, lanes 1 and 2 , top panel), or when protein was immunoprecipitated with anti-phospho-eIF4GI antibody (Figure 3A, lanes 5 and 6 , top panel). In contrast, anti-VPg antibody exclusively precipitated full-length eIF4GI. If the interaction between VPg and eIF4GI is direct, these data suggest a binding site for VPg could reside in the N-terminal twothirds of eIF4GI, since the phospho-eIF4GI antibody recognizes the $\mathrm{C}$ terminal third of the protein. If the interaction between VPg and eIF4GI is indirect, full length eIF4GI could have been brought down by a potential direct interaction between VPg and eIF4E because an eIF4E binding site resides in the $\mathrm{N}$ terminus of eIF4GI [17].

Finally, the presence of identical degradation products in both mock and infected cell lysates suggests that, in contrast to FCV infections [18], eIF4GI is not detectably cleaved during MNV-1 infection. Although we have not performed extensive analysis of eIF4GI cleavage, these data are consistent with reports that recombinant norovirus 3C protease (MD-145 strain) does not cleave eIF4GI in vitro [19]. This degradation pattern was not observed when mock and infected cell lysates were probed with antibody to total eIF4GI (Figure 3B). The reason for this discrepancy is not entirely clear, but we have observed some variability in the stability of eIF4GI in RAW 264.7 cells lysates. The degradation products also could be C-terminal fragments of the protein not recognized by the eIF4GI antibody made to an $\mathrm{N}$-terminal peptide that was used to detect the full-length protein.

Goodfellow and co-workers recently reported that FCV VPg co-precipitates eIF4E from infected cells, and that this interaction is necessary for translation of FCV VPg-linked RNA in vitro [6]. To determine if MNV-1 VPg was associated with eIF4E in infected cells, we immunoprecipitated VPg from infected cell lysates and probed samples by immunoblot for eIF4E. eIF4E was found in similar quantities in mock and infected cell lysates in the absence of antibody (Figure 3A, lanes 1 and 2, bottom panel), and co-precipitated with VPg from infected cells (Figure 3A, lanes 3 and 4, bottom panel). These data indicate that MNV-1 VPg and eIF4E interact in infected cells. Further studies are necessary to determine if this interaction with MNV-1 VPg is direct, or if eIF4E is detected as a result of interactions with other components of eIF4F.

\section{Discussion}

We and others put forth the idea that VPg functions in translation initiation on VPg-linked RNA through interactions with eIFs $[3,5,6,20]$. The data presented here provide experimental evidence of interactions between VPg and eIFs in norovirus infected cells, and strengthen the assertion that the interactions previously defined for NV VPg by cell-free assays are relevant and functional.

eIF3, eIF4GI, and eIF4E were detected in GST pull-down eluates of both NV VPg [5] and MNV-1 VPg (this study), and eIF4GI and eIF4E were found in co-immunoprecipitates with VPg from MNV-1 infected cells. While these assays do not prove a direct interaction between VPg and these translation initiation factors, they strongly suggest VPg interacts with one or more components of the eIF4F complex. These observations lend support to previous suggestions that VPg is a protein cap analogue with respect to binding interactions with eIF4F. We further suggest that the role of VPg in ribosome recruitment is more complex than providing a target for eIF4F binding. VPg is a $15 \mathrm{kD}$ protein covalently linked to the 5' end of an RNA that has a 10 nucleotide $5^{\prime}$ UTR. If VPg functions simply as a protein cap, then by analogy to capped mRNA, translation initiation at nucleotide 11 would be inefficient [21]. Moreover, direct interactions between VPg and eIF3 [5] point to the potential involvement of VPg in $43 \mathrm{~S}$ pre-initiation complex recruitment. Sequence conservation between VPg and initiation factor eIF1A, a factor important in AUG codon recognition and ternary complex recruitment [22], has been noted for NV VPg [23], and a similar degree of conservation exists between MNV-1 VPg and eIF1A (data not shown). We have suggested a model of translation initiation on NV VPg-linked RNA that we now extend to MNV-1, whereby a direct interaction between VPg and eIF3, and likely components of eIF4F, positions the ribosome at the initiator AUG without ribosome scanning. This model implies numerous concerted interactions between norovirus VPg and eIFs, including the $40 \mathrm{~S}$ ribosome, additional to the interactions reported so far. 
Studies with animal calicivirus strains in the Vesivirus genus of the family, particularly FCV, have contributed a large body of data on mechanisms of calicivirus replication $[19,24-26]$. These data provide a good foundation on which to build models of norovirus replication by analogy. However, these analogies have limitations, largely due to the genetic divergence between animal caliciviruses and noroviruses. For example, FCV VPg and NV VPg share only $18 \%$ amino acid identity, and thus far we and others [6] have been unable to detect interactions between FCV VPg and eIF3 or eIF4GI in GST pull-down assays. We have not performed extensive binding studies with FCV VPg to determine what interactions may occur that are different from those observed with the norovirus VPg, but it is probable that specific interactions and mechanistic details differ between the animal caliciviruses and noroviruses. These observations exemplify the importance of MNV-1 as a molecular model for human noroviruses, as interactions between MNV-1 VPg and eIFs precisely mimic those reported for NV VPg.

\section{Methods}

\section{RAW 264.7 Cells}

RAW 264.7 cells (ATCC TIB-71) were maintained in DMEM containing 10\% FBS (Atlanta Biologicals), $10 \mathrm{mM}$ Hepes, $4 \mathrm{mM}$ L-glutamine, $4.5 \mathrm{~g} / \mathrm{L}$ glucose, and $1.5 \mathrm{~g} / \mathrm{L}$ sodium bicarbonate.

\section{Construction of GST-MNV-I VPg and GST pull-down assays}

MNV-1 VPg was amplified from pSPORT-T7-MNV-1 (gift from $H$. Virgin, Washington University School of Medicine) using the primers MNV-VPg/BamHI(+) 5'-cgcggatccggaaagaagggcaagaac-3' and MNV-VPg/XhoI(-) 5'ccgctcgagctcaaagttgatcttctcg-3'. Restriction sites for cloning are underlined. Reactions were assembled using the KOD kit (Novagen) according to instructions provided by the manufacturer. Amplification conditions consisted of 25 cycles of $98^{\circ} \mathrm{C}$ for 15 seconds, $58^{\circ} \mathrm{C}$ for 3 seconds, and $72^{\circ} \mathrm{C}$ for 5 seconds. The amplification product was cloned into pGEX-4-T1 (GE Amersham Biosciences) using BamHI and XhoI restriction enzymes. Expression and purification of GST-MNV-VPg, as well as pull-down assays using RAW 264.7 cell lysates, were performed as described previously [5].

\section{Immunoprecipitations}

RAW 264.7 cells were grown to $90 \%$ confluence in $10 \mathrm{~cm}$ dishes, and infected with MNV-1 for 17 hours at a multiplicity of infection of $1.5 \mathrm{pfu} /$ cell. Cells were harvested in $1 \mathrm{~mL}$ of cold medium and pelleted for 5 minutes at 3000 $\times g$ at $4{ }^{\circ} \mathrm{C}$. Pellets were washed twice in cold phosphatebuffered saline, and lysed by incubation on ice for 30 minutes in $500 \mu \mathrm{L}$ IP buffer containing $20 \mathrm{mM}$ Tris- $\mathrm{HCl}$ $\mathrm{pH} 7.5,50 \mathrm{mM} \mathrm{NaCl}, 0.1 \% \mathrm{NP}-40,10 \mathrm{mM} \beta$-glycerophos- phate, $5 \mathrm{mM} \mathrm{NaF}, 2.5 \%$ glycerol, and one mini protease inhibitor cocktail tablet (Roche Biochemicals). Lysates were clarified by centrifugation for 5 minutes at 10,000 $\times$ $g$ at $4{ }^{\circ} \mathrm{C}$. Ten $\mu \mathrm{l}(2 \%)$ of each sample was retained as controls and not subjected to immunoprecipitation. The remaining lysate was incubated with the indicated antibody and rotated gently for two hours at $4{ }^{\circ} \mathrm{C}$. Anti-phospho-eIF4GI (Cell Signaling Technologies) was used at a dilution of 1:100 and anti-MNV-1 VPg (provided by Kim Green, NIAID) was used at a dilution of 1:1000. Thirty $\mu \mathrm{l}$ of Gamma Bind Plus Sepharose beads (GE Amersham Biosciences) prepared in IP buffer were added, and complexes were rotated for one hour at $4{ }^{\circ} \mathrm{C}$. Beads were collected by centrifugation for 2 minutes at $500 \times g$ at $4{ }^{\circ} \mathrm{C}$, and then washed four times with wash buffer containing $20 \mathrm{mM}$ Tris-HCl pH 7.5, $50 \mathrm{mM} \mathrm{NaCl}$, and 0.1\% NP-40. Complexes were eluted from the beads by incubation on ice for 10 minutes in $25 \mu$ l elution buffer $(0.75 \% \mathrm{wt} / \mathrm{vol}$ glycine in water, $\mathrm{pH}$ 2.2). The beads were collected for 2 minutes at $500 \times g$ at $4{ }^{\circ} \mathrm{C}$. Supernatants were transferred to fresh tubes containing $0.9 \mu \mathrm{l} 2 \mathrm{M}$ Tris base.

\section{Western immunoblots}

Immunoprecipitation and pull-down samples were subjected to SDS-PAGE and transferred to nitrocellulose, blocked in $10 \%$ nonfat dry milk and incubated with the indicated antibody overnight at room temperature. Antibodies used were anti-phospho-eIF4GI at 1:1000, anti-S6 ribosomal protein and anti-eIF4E (both from Cell Signaling Technologies) at 1:1000, anti-eIF3 (kindly provided by John Hershey, UC Davis) at 1:2000, anti-eIF4GI (N-20, Santa Cruz Biotechnology) at 1:1000, and anti-MNV-1 VPg at 1:2000. Appropriate horseradish peroxidase-conjugated secondary antibodies were used at dilutions of 1:3000. Blots were developed with ECL chemiluminescent substrate (GE Amersham Biosciences) and exposed to film for similar lengths of time.

\section{Competing interests}

The author(s) declare that they have no competing interests.

\section{Acknowledgements}

We thank Herbert W Virgin (Washington University in St Louis) for generously providing reagents and advice on culture and manipulation of MNVI. We thank Kim Green (NIAID) for antiserum to MNV-I VPg. This work was supported by PHS grant Al-43450 to MEH, PHS grant P20 RR020 I 85, and by the Montana Agricultural Experiment Station. KFD was partially supported by the Kopriva Fellowship and USDA National Needs Program. CEW was supported by NIH Grant U54 Al057I60 to the Midwest Regional Center of Excellence for Biodefense and Emerging Infectious Disease Research.

\section{References}

I. Green KY: Human caliciviruses. In Fields Virology Edited by: Knipe DM, Howley PM. Lippincott Williams \& Wilkins, Philadelphia, PA; 200I:84I-874. 
2. Burroughs JN, Brown F: Presence of a covalently linked protein on calicivirus RNA. J Gen Virol 1978, 4 I:443-446.

3. Herbert TP, Brierley I, Brown TD: Identification of a protein linked to the genomic and subgenomic mRNAs of feline calicivirus and its role in translation. J Gen Virol 1997, 78(Pt 5): $1033-1040$.

4. Sosnovtsev S, Green KY: RNA transcripts derived from a cloned full-length copy of the feline calicivirus genome do not require VpG for infectivity. Virology 1995, 21 0:383-390.

5. Daughenbaugh K, Fraser CSHJW, Hardy ME: The genome linked protein VPg of the Norwalk virus binds elF3, suggesting its role in translation initiation complex recruitment. $E M B O$ 2003, 22:2852-2859.

6. Goodfellow I, Chaudhry Y, Gioldasi I, Gerondopoulos A, Natoni A Labrie L, Laliberte JF, Roberts L: Calicivirus translation initiation requires an interaction between VPg and elF $4 \mathrm{E}$. EMBO Rep 2005, 6:968-972.

7. Hershey JWB, Merrick WC: The pathway and mechanism of initiation of protein synthesis. Translational Control of Gene Expression. Cold Sring Harbor Laboratory Press, Cold Spring Harbor; 2000:33-88

8. Hentze MW: elF4G: a multipurpose ribosome adapter? Science 1997, 275:500-50I.

9. Gingras AC, Raught B, Sonenberg N: elF4 initiation factors: effectors of mRNA recruitment to ribosomes and regulators of translation. Annu Rev Biochem 1999, 68:913-963.

10. Sarnow P, Cevallos RC, Jan E: Takeover of host ribosomes by divergent IRES elements. Biochem Soc Trans 2005, 33: | 479- I 482

II. Hellen CU, Sarnow P: Internal ribosome entry sites in eukaryotic mRNA molecules. Genes Dev 200I, 15:1593-1612.

12. Karst SM, Wobus CE, Lay M, Davidson J, Virgin HW: STATIdependent innate immunity to a Norwalk-like virus. Science 2003, 299:1575-1578

13. Wobus CE, Karst SM, Thackray LB, Chang KO, Sosnovtsev SV, Belliot G, Krug A, Mackenzie JM, Green KY, Virgin HW: Replication of Norovirus in cell culture reveals a tropism for dendritic cells and macrophages. PLoS Biol 2004, 2:e432.

14. Meyer LJ, Milburn SC, Hershey JW: Immunochemical characterization of mammalian protein synthesis initiation factors. Biochemistry 1982, $21: 4206-4212$.

15. Fontaine-Rodriguez EC, Taylor TJ, Olesky M, Knipe DM: Proteomics of herpes simplex virus infected cell protein 27: association with translation initiation factors. Virology 2004, 330:487-492.

16. Raught B, Gingras AC, Gygi SP, Imataka H, Morino S, Gradi A, Aebersold $R$, Sonenberg N: Serum-stimulated, rapamycin-sensitive phosphorylation sites in the eukaryotic translation initiation factor 4GI. EMBO I 2000, 19:434-444.

17. Mader $\mathrm{S}$, Lee $\mathrm{H}$, Pause $\mathrm{A}$, Sonenberg $\mathrm{N}$ : The translation initiation factor elF-4E binds to a common motif shared by the translation factor elF-4 gamma and the translational repressors 4E-binding proteins. Mol Cell Biol 1995, I 5:4990-4997.

18. Willcocks MM, Carter MJ, Roberts LO: Cleavage of eukaryotic initiation factor elF4G and inhibition of host-cell protein synthesis during feline calicivirus infection. J Gen Virol 2004, 85: II $25-1130$

19. Kuyumcu-Martinez M, Belliot G, Sosnovtsev SV, Chang KO, Green $\mathrm{KY}$, Lloyd RE: Calicivirus 3C-like proteinase inhibits cellular translation by cleavage of poly(A)-binding protein. J Virol 2004, 78:8I72-8I82

20. Dunham DM, Jiang X, Berke T, Smith AW, Matson DO: Genomic mapping of a calicivirus VPg. Arch Virol 1998, 143:242I-2430.

21. Kozak M: A short leader sequence impairs the fidelity of initiation by eukaryotic ribosomes. Gene Expr 1991, I: I II-II5.

22. Pestova TV, Kolupaeva VG: The roles of individual eukaryotic translation initiation factors in ribosomal scanning and initiation codon selection. Genes Dev 2002, 16:2906-2922.

23. Sosnovtsev SV, Green KY: Identification and Genomic Mapping of the ORF3 and VPg Proteins in Feline Calicivirus Virions. Virology 2000, 277: 193-203.

24. Green KY, Mory A, Fogg MH, Weisberg A, Belliot G, Wagner M, Mitra T, Ehrenfeld E, Cameron CE, Sosnovtsev SV: Isolation of enzymatically active replication complexes from feline calicivirus-infected cells. J Virol 2002, 76:8582-8595.
25. Sosnovtser SV, Garfield M, Green KY: Processing map and essential cleavage sites of the nonstructural polyprotein encoded by ORFI of the feline calicivirus genome. 2002.

26. Sosnovtsey SV, Belliot G, Chang KO, Onwudiwe O, Green KY: Feline calicivirus VP2 is essential for the production of infectious virions. J Virol 2005, 79:4012-4024.
Publish with BioMed Central and every scientist can read your work free of charge

"BioMed Central will be the most significant development for disseminating the results of biomedical research in our lifetime. "

Sir Paul Nurse, Cancer Research UK

Your research papers will be:

- available free of charge to the entire biomedical community

- peer reviewed and published immediately upon acceptance

- cited in PubMed and archived on PubMed Central

- yours - you keep the copyright 